\title{
ON EIGHT SOLVABLE SYSTEMS OF DIFFERENCE EQUATIONS IN TERMS OF GENERALIZED PADOVAN SEQUENCES
}

\author{
M. KARA AND Y. YAZLIK
}

Received 17 February, 2020

\begin{abstract}
In this study we show that the systems of difference equations

$$
x_{n+1}=f^{-1}\left(a f\left(p_{n-1}\right)+b f\left(q_{n-2}\right)\right), y_{n+1}=f^{-1}\left(a f\left(r_{n-1}\right)+b f\left(s_{n-2}\right)\right),
$$

for $n \in \mathbb{N}_{0}$, where the sequences $p_{n}, q_{n}, r_{n}$ and $s_{n}$ are some of the sequences $x_{n}$ and $y_{n}$, $f: D_{f} \longrightarrow \mathbb{R}$ is a " $1-1$ " continuous function on its domain $D_{f} \subseteq \mathbb{R}$, initial values $x_{-j}, y_{-j}$, $j \in\{0,1,2\}$, are arbitrary real numbers in $D_{f}$ and the parameters $a, b$ are arbitrary complex numbers, with $b \neq 0$, can be explicitly solved in terms of generalized Padovan sequences. Some analytical examples are given to demonstrate the theoretical results.
\end{abstract}

2010 Mathematics Subject Classification: 39A10; 39A20

Keywords: system of difference equations, solution of explicit form, Padovan number

\section{INTRODUCTION}

Firstly, recall that $\mathbb{N}, \mathbb{N} 0, \mathbb{Z}, \mathbb{R}, \mathbb{C}$, stand for natural, non-negative integer, integer, real and complex numbers, respectively. If $m, n \in \mathbb{Z}, m \leq n$ the notation $i=\overline{m, n}$ stands for $\{i \in \mathbb{Z}: m \leq i \leq n\}$.

Difference equations for which the solutions can be constructed explicitly are useful due to numerous applications. As particular, difference equations related to Fibonacci, Lucas, Padovan, Tetranacci, Horadam, Pell, Jacobsthal, and Jacobsthal-Lucas sequences and their generalizations are of much interest. Many related references can be found, for example, in [5-7, 10,11, 17-19,21,23].

The equation

$$
x_{n+1}=\frac{a x_{n-l} x_{n-k}}{b x_{n-p} \pm c x_{n-q}}, n \in \mathbb{N}_{0},
$$

where the initial conditions are arbitrary positive real numbers, $k, l, p, q$ are nonnegative integers and $a, b, c$ are positive constants, is one of the difference equations whose solutions are associated with number sequences. Positive solutions of concrete special cases of equation (1.1) have been studied by several authors. For the first time, Elabbasy et al., in [6,7], obtained positive solutions of some special cases of equation (1.1) by using induction principle. In addition, they didn't give theoretical 
explanation of how solutions were obtained. One of the special cases is

$$
x_{n+1}=\frac{x_{n-1} x_{n-2}}{x_{n-1}+x_{n-2}}, n \in \mathbb{N}_{0},
$$

whose solutions are associated with the well known Padovan numbers in literature. Moreover, the multi-dimensional expansion of the concrete some special cases of equation (1.1) can be seen in the literature (see, for example, [2-4, 8, 9, 13, 14, 24]). Another equation

$$
x_{n+1}=a+\frac{b}{x_{n}}+\frac{c}{x_{n} x_{n-1}}, n \in \mathbb{N}_{0},
$$

where the parameters $a, b, c$ and initial values $x_{-1}$ and $x_{0}$ are complex numbers and $c \neq 0$, which is one of these equations. The solutions of equation (1.3) are associated with number sequences, has been studied in [16]. Unlike the method used to obtain solutions of some special cases of (1.1), by using convenient transformation the equation in (1.3) reduce to the next third-order linear difference equation with constant coefficients

$$
x_{n+1}=a x_{n}+b x_{n-1}+c x_{n-2}, \quad n \in \mathbb{N}_{0},
$$

which has actually the general solution

$$
x_{n}=x_{0} S_{n}+x_{-1}\left(S_{n+1}-a S_{n}\right)+c x_{-2} S_{n-1}, \quad n \in \mathbb{N}_{0},
$$

where $\left(S_{n}\right)_{n \geq-2}$ of equation (1.4) satisfying the initial values $S_{-2}=S_{-1}=0, S_{0}=1$. Quite recently in [15], among other things, a generalization of (1.4) is treated as

$$
x_{n}=f^{-1}\left(a f\left(x_{n-1}\right)+b f\left(x_{n-2}\right)+c f\left(x_{n-3}\right)\right), n \in \mathbb{N}_{0},
$$

where $f: D_{f} \longrightarrow \mathbb{R}$ is a " $1-1$ " continuous function on its domain $D_{f} \subseteq \mathbb{R}$, parameters $a, b, c$ and the initial values $x_{-3}, x_{-2}$ and $x_{-1}$ are real numbers. In addition, the authors obtained the solution of the equation (1.6) in relation to the solution given in (1.5).

On the other hand, one of the popular topics for system of difference equations is also symmetric and close-to-symmetric systems such as

$$
x_{n+1}=g\left(p_{n-k}, q_{n-l}\right), y_{n+1}=g\left(r_{n-k}, s_{n-l}\right), n \in \mathbb{N}_{0},
$$

where the sequences $p_{n}, q_{n}, r_{n}, s_{n}$ are some of the sequences $x_{n}$ and $y_{n}$ and $k, l$ are fixed natural numbers. There are studies related to some special cases of the system (1.7) (see, for example, [1, 12, 20, 22]).

Motivated by this line of investigations, here we show that the systems of difference equations

$$
x_{n+1}=f^{-1}\left(a f\left(p_{n-1}\right)+b f\left(q_{n-2}\right)\right), y_{n+1}=f^{-1}\left(a f\left(r_{n-1}\right)+b f\left(s_{n-2}\right)\right),
$$

for $n \in \mathbb{N}_{0}$, where the sequences $p_{n}, q_{n}, r_{n}$ and $s_{n}$ are some of the sequences $x_{n}$ and $y_{n}, f: D_{f} \longrightarrow \mathbb{R}$ is a " $1-1$ " continuous function on its domain $D_{f} \subseteq \mathbb{R}$, the initial values $x_{-j}, y_{-j}, j \in\{0,1,2\}$ are arbitrary real numbers and the parameters and $a, b$ 
are arbitrary complex numbers, can be solved. To do this, we will use the solutions given in (1.5) and the solutions obtained by rearranging these solutions. In this way we also give analytical examples for the general solution of special cases of system (1.8).

\section{MAin Results}

In this section, we consider the eight special cases of systems (1.8), where the sequences $p_{n}, q_{n}, r_{n}, s_{n}$ are some of the sequences $x_{n}$ and $y_{n}$, for $n \geq-2$, and initial values $x_{-j}, y_{-j}, j \in\{0,1,2\}$, are arbitrary real numbers.

2.1. Case 1: $p_{n}=x_{n}, q_{n}=x_{n}, r_{n}=y_{n}, s_{n}=y_{n}$

In this case, system (1.8) is expressed as

$$
x_{n+1}=f^{-1}\left(a f\left(x_{n-1}\right)+b f\left(x_{n-2}\right)\right), y_{n+1}=f^{-1}\left(a f\left(y_{n-1}\right)+b f\left(y_{n-2}\right)\right) \text {, }
$$

for $n \in \mathbb{N}_{0}$. Since $f$ is " $1-1$ ", from (2.1)

$$
f\left(x_{n+1}\right)=a f\left(x_{n-1}\right)+b f\left(x_{n-2}\right), f\left(y_{n+1}\right)=a f\left(y_{n-1}\right)+b f\left(y_{n-2}\right),
$$

for $n \in \mathbb{N}_{0}$. By using the change of variables

$$
f\left(x_{n}\right)=u_{n} \text {, and } f\left(y_{n}\right)=v_{n}, n \geq-2,
$$

system (2.2) is transformed to the following one

$$
u_{n+1}=a u_{n-1}+b u_{n-2}, \quad v_{n+1}=a v_{n-1}+b v_{n-2},
$$

for $n \in \mathbb{N}_{0}$. By taking $a=0, b=a, c=b$ in (1.4) and $S_{n}=J_{n+1}$, for all $n \geq-2$, which is called generalized Padovan sequence, in (1.5), the solutions to equations in (2.4) are given by

$$
\begin{aligned}
& u_{n}=u_{0} J_{n+1}+u_{-1} J_{n+2}+b u_{-2} J_{n}, \\
& v_{n}=v_{0} J_{n+1}+v_{-1} J_{n+2}+b v_{-2} J_{n},
\end{aligned}
$$

for $n \in \mathbb{N}_{0}$. From (2.3), (2.5) and (2.6), it follows that the general solution to system (2.2) is given by

$$
\begin{aligned}
& x_{n}=f^{-1}\left(f\left(x_{0}\right) J_{n+1}+f\left(x_{-1}\right) J_{n+2}+b f\left(x_{-2}\right) J_{n}\right), n \geq-2, \\
& y_{n}=f^{-1}\left(f\left(y_{0}\right) J_{n+1}+f\left(y_{-1}\right) J_{n+2}+b f\left(y_{-2}\right) J_{n}\right), n \geq-2 .
\end{aligned}
$$

2.2. Case 2: $p_{n}=x_{n}, q_{n}=x_{n}, r_{n}=x_{n}, s_{n}=x_{n}$

In this case, system (1.8) becomes

$$
x_{n+1}=f^{-1}\left(a f\left(x_{n-1}\right)+b f\left(x_{n-2}\right)\right), y_{n+1}=f^{-1}\left(a f\left(x_{n-1}\right)+b f\left(x_{n-2}\right)\right) \text {, }
$$

for $n \in \mathbb{N}_{0}$. It should be first note that from the equations in (2.9) it immediately follows that $x_{n}=y_{n}$, for all $n \in \mathbb{N}$. From (2.7), the general solution to system (2.9) is

$$
x_{n}=y_{n}=f^{-1}\left(f\left(x_{0}\right) J_{n+1}+f\left(x_{-1}\right) J_{n+2}+b f\left(x_{-2}\right) J_{n}\right), n \in \mathbb{N} \text {. }
$$


2.3. Case 3: $p_{n}=y_{n}, q_{n}=y_{n}, r_{n}=y_{n}, s_{n}=y_{n}$

In this case, we obtain the system

$$
x_{n+1}=f^{-1}\left(a f\left(y_{n-1}\right)+b f\left(y_{n-2}\right)\right), y_{n+1}=f^{-1}\left(a f\left(y_{n-1}\right)+b f\left(y_{n-2}\right)\right) \text {, }
$$

for $n \in \mathbb{N}_{0}$, which is an analogue of the system (2.9). By interchanging the variables $x_{n}$ and $y_{n}$, the system in (2.9) is transformed into (2.11). So, by interchanging $x_{j}$ and $y_{j}$ for $j \in\{0,1,2\}$, the formula in (2.10) is transformed into the formula

$$
x_{n}=y_{n}=f^{-1}\left(f\left(y_{0}\right) J_{n+1}+f\left(y_{-1}\right) J_{n+2}+b f\left(y_{-2}\right) J_{n}\right), n \in \mathbb{N} .
$$

2.4. Case 4: $p_{n}=x_{n}, q_{n}=x_{n}, r_{n}=y_{n}, s_{n}=x_{n}$

In this case, system (1.8) is written as in the form

$$
x_{n+1}=f^{-1}\left(a f\left(x_{n-1}\right)+b f\left(x_{n-2}\right)\right), y_{n+1}=f^{-1}\left[a f\left(y_{n-1}\right)+b f\left(x_{n-2}\right)\right] \text {, }
$$

for $n \in \mathbb{N}_{0}$. Since $f$ is " $1-1$ ", from (2.13)

$$
f\left(x_{n+1}\right)=a f\left(x_{n-1}\right)+b f\left(x_{n-2}\right), f\left(y_{n+1}\right)=a f\left(y_{n-1}\right)+b f\left(x_{n-2}\right),
$$

for $n \in \mathbb{N}_{0}$. By using the change of variables

$$
f\left(x_{n}\right)=u_{n}, n \geq-2, \text { and } f\left(y_{n}\right)=v_{n}, n \geq-1,
$$

system (2.14) is transformed to the following one

$$
u_{n+1}=a u_{n-1}+b u_{n-2}, \quad v_{n+1}=a v_{n-1}+b u_{n-2},
$$

for $n \in \mathbb{N}_{0}$. From (2.5), we can write the solution of the first equation in (2.16) as

$$
u_{n}=u_{0} J_{n+1}+u_{-1} J_{n+2}+b u_{-2} J_{n}, n \in \mathbb{N}_{0} .
$$

By subtracting the second one from the first equations in (2.16), we have

$$
u_{n+1}-v_{n+1}=a\left(u_{n-1}-v_{n-1}\right), n \in \mathbb{N}_{0} .
$$

From (2.18) we see that the sequence $\left(u_{n}-v_{n}\right)_{n \geq-1}$ satisfies the difference equation

$$
w_{n}=a w_{n-2}, n \geq 1,
$$

from which it follows that

$$
u_{2 n+i}-v_{2 n+i}=a^{n+1}\left(u_{i-2}-v_{i-2}\right),
$$

for $n \in \mathbb{N}_{0}, i \in\{1,2\}$.

From (2.17) and (2.20), we get

$$
\begin{aligned}
v_{2 n+i} & =u_{2 n+i}-a^{n+1} u_{i-2}+a^{n+1} v_{i-2}, \\
& =u_{0} J_{2 n+i+1}+u_{-1} J_{2 n+i+2}+b u_{-2} J_{2 n+i}-a^{n+1} u_{i-2}+a^{n+1} v_{i-2},
\end{aligned}
$$

for $n \in \mathbb{N}_{0}, i \in\{1,2\}$.

Employing (2.17) and (2.21) in (2.15) and after some calculation, we obtain

$$
x_{n}=f^{-1}\left(f\left(x_{0}\right) J_{n+1}+f\left(x_{-1}\right) J_{n+2}+b f\left(x_{-2}\right) J_{n}\right), n \geq-2 \text {, }
$$




$$
\begin{aligned}
y_{2 n+1}= & f^{-1}\left(f\left(x_{0}\right) J_{2 n+2}+f\left(x_{-1}\right)\left(J_{2 n+3}-a^{n+1}\right)+b f\left(x_{-2}\right) J_{2 n+1}\right. \\
& \left.+a^{n+1} f\left(y_{-1}\right)\right), n \geq-1, \\
y_{2 n+2}= & f^{-1}\left(f\left(x_{0}\right)\left(J_{2 n+3}-a^{n+1}\right)+f\left(x_{-1}\right) J_{2 n+4}+b f\left(x_{-2}\right) J_{2 n+2}\right. \\
& \left.+a^{n+1} f\left(y_{0}\right)\right), n \geq-1 .
\end{aligned}
$$

2.5. Case 5: $p_{n}=x_{n}, q_{n}=y_{n}, r_{n}=y_{n}, s_{n}=y_{n}$

In this case, system (1.8) is expressed as

$$
x_{n+1}=f^{-1}\left(a f\left(x_{n-1}\right)+b f\left(y_{n-2}\right)\right), y_{n+1}=f^{-1}\left(a f\left(y_{n-1}\right)+b f\left(y_{n-2}\right)\right),
$$

for $n \in \mathbb{N}_{0}$. Note that system (2.25) is obtained from equations (2.13) by interchanging the letters $x$ and $y$, and hence all the statements concerning solutions to the equations follow from the corresponding statements in Case 4.

The general solution to the system $(2.25)$ is given

$$
\begin{aligned}
x_{2 n+1}= & f^{-1}\left(f\left(y_{0}\right) J_{2 n+2}+f\left(y_{-1}\right)\left(J_{2 n+3}-a^{n+1}\right)+b f\left(y_{-2}\right) J_{2 n+1}\right. \\
& \left.+a^{n+1} f\left(x_{-1}\right)\right), n \geq-1, \\
x_{2 n+2}= & f^{-1}\left(f\left(y_{0}\right)\left(J_{2 n+3}-a^{n+1}\right)+f\left(y_{-1}\right) J_{2 n+4}+b f\left(y_{-2}\right) J_{2 n+2}\right. \\
& \left.+a^{n+1} f\left(x_{0}\right)\right), n \geq-1, \\
y_{n}= & f^{-1}\left(f\left(y_{0}\right) J_{n+1}+f\left(y_{-1}\right) J_{n+2}+b f\left(y_{-2}\right) J_{n}\right), n \geq-2 .
\end{aligned}
$$

2.6. Case 6: $p_{n}=y_{n}, q_{n}=y_{n}, r_{n}=x_{n}, s_{n}=x_{n}$

In this case, we obtain the system

$$
x_{n+1}=f^{-1}\left(a f\left(y_{n-1}\right)+b f\left(y_{n-2}\right)\right), y_{n+1}=f^{-1}\left(a f\left(x_{n-1}\right)+b f\left(x_{n-2}\right)\right),
$$

for $n \in \mathbb{N}_{0}$. Since $f$ is " $1-1$ ", from (2.29)

$$
f\left(x_{n+1}\right)=a f\left(y_{n-1}\right)+b f\left(y_{n-2}\right), f\left(y_{n+1}\right)=a f\left(x_{n-1}\right)+b f\left(x_{n-2}\right),
$$

for $n \in \mathbb{N}_{0}$. By using the change of variables

$$
f\left(x_{n}\right)=u_{n}, \text { and } f\left(y_{n}\right)=v_{n}, n \geq-2,
$$

system (2.30) is transformed to the following one

$$
u_{n+1}=a v_{n-1}+b v_{n-2}, \quad v_{n+1}=a u_{n-1}+b u_{n-2}, n \in \mathbb{N}_{0} .
$$

By summing the equations in (2.32) we get

$$
u_{n+1}+v_{n+1}=a\left(u_{n-1}+v_{n-1}\right)+b\left(u_{n-2}+v_{n-2}\right), n \in \mathbb{N}_{0},
$$

whereas by subtracting the second one from the first, we have

$$
u_{n+1}-v_{n+1}=-a\left(u_{n-1}-v_{n-1}\right)-b\left(u_{n-2}-v_{n-2}\right), n \in \mathbb{N}_{0} .
$$


From (2.5), we can write the solution of equation (2.33) as

$$
u_{n}+v_{n}=\left(u_{0}+v_{0}\right) J_{n+1}+\left(u_{-1}+v_{-1}\right) J_{n+2}+b\left(u_{-2}+v_{-2}\right) J_{n},
$$

for $n \geq-2$. On the other hand, by taking $a=0, b=-a, c=-b$ in (1.4) and $S_{n}=(-1)^{n} J_{n+1}^{\prime}$, for all $n \geq-2$, which is called generalized Padovan sequence, in (1.5), from (2.34), we also have

$$
u_{n}-v_{n}=(-1)^{n}\left(\left(u_{0}-v_{0}\right) J_{n+1}^{\prime}-\left(u_{-1}-v_{-1}\right) J_{n+2}^{\prime}+b\left(u_{-2}-v_{-2}\right) J_{n}^{\prime}\right),
$$

for $n \geq-2$. From (2.36) we obtain

$$
u_{2 n}-v_{2 n}=\left(u_{0}-v_{0}\right) J_{2 n+1}^{\prime}-\left(u_{-1}-v_{-1}\right) J_{2 n+2}^{\prime}+b\left(u_{-2}-v_{-2}\right) J_{2 n}^{\prime},
$$

for $n \geq-1$. From (2.35)

$$
u_{2 n}+v_{2 n}=\left(u_{0}+v_{0}\right) J_{2 n+1}+\left(u_{-1}+v_{-1}\right) J_{2 n+2}+b\left(u_{-2}+v_{-2}\right) J_{2 n},
$$

for $n \geq-1$. By summing the equations (2.37) and (2.38) we get

$$
\begin{aligned}
u_{2 n}= & \frac{\left(J_{2 n+1}+J_{2 n+1}^{\prime}\right) u_{0}+\left(J_{2 n+1}-J_{2 n+1}^{\prime}\right) v_{0}+\left(J_{2 n+2}-J_{2 n+2}^{\prime}\right) u_{-1}}{2} \\
& +\frac{\left(J_{2 n+2}+J_{2 n+2}^{\prime}\right) v_{-1}+b\left(J_{2 n}+J_{2 n}^{\prime}\right) u_{-2}+b\left(J_{2 n}-J_{2 n}^{\prime}\right) v_{-2}}{2},
\end{aligned}
$$

for $n \geq-1$. By subtracting equation (2.37) from equation (2.38), we have

$$
\begin{aligned}
v_{2 n}= & \frac{\left(J_{2 n+1}-J_{2 n+1}^{\prime}\right) u_{0}+\left(J_{2 n+1}+J_{2 n+1}^{\prime}\right) v_{0}+\left(J_{2 n+2}+J_{2 n+2}^{\prime}\right) u_{-1}}{2} \\
& +\frac{\left(J_{2 n+2}-J_{2 n+2}^{\prime}\right) v_{-1}+b\left(J_{2 n}-J_{2 n}^{\prime}\right) u_{-2}+b\left(J_{2 n}+J_{2 n}^{\prime}\right) v_{-2}}{2},
\end{aligned}
$$

for $n \geq-1$. From (2.36) we have

$$
u_{2 n+1}-v_{2 n+1}=-\left(u_{0}-v_{0}\right) J_{2 n+2}^{\prime}+\left(u_{-1}-v_{-1}\right) J_{2 n+3}^{\prime}-b\left(u_{-2}-v_{-2}\right) J_{2 n+1}^{\prime} \text {, }
$$

for $n \geq-1$. From (2.35)

$$
u_{2 n+1}+v_{2 n+1}=\left(u_{0}+v_{0}\right) J_{2 n+2}+\left(u_{-1}+v_{-1}\right) J_{2 n+3}+b\left(u_{-2}+v_{-2}\right) J_{2 n+1},
$$

for $n \geq-1$. By summing the equations (2.41) and (2.42) we get

$$
\begin{aligned}
u_{2 n+1}= & \frac{\left(J_{2 n+2}-J_{2 n+2}^{\prime}\right) u_{0}+\left(J_{2 n+2}+J_{2 n+2}^{\prime}\right) v_{0}+\left(J_{2 n+3}+J_{2 n+3}^{\prime}\right) u_{-1}}{2} \\
& +\frac{\left(J_{2 n+3}-J_{2 n+3}^{\prime}\right) v_{-1}+b\left(J_{2 n+1}-J_{2 n+1}^{\prime}\right) u_{-2}}{2}+\frac{b\left(J_{2 n+1}+J_{2 n+1}^{\prime}\right) v_{-2}}{2},
\end{aligned}
$$

for $n \geq-1$. By subtracting equation (2.41) from equation (2.42), we have

$$
v_{2 n+1}=\frac{\left(J_{2 n+2}+J_{2 n+2}^{\prime}\right) u_{0}+\left(J_{2 n+2}-J_{2 n+2}^{\prime}\right) v_{0}+\left(J_{2 n+3}-J_{2 n+3}^{\prime}\right) u_{-1}}{2}
$$




$$
+\frac{\left(J_{2 n+3}+J_{2 n+3}^{\prime}\right) v_{-1}+b\left(J_{2 n+1}+J_{2 n+1}^{\prime}\right) u_{-2}}{2}+\frac{b\left(J_{2 n+1}-J_{2 n+1}^{\prime}\right) v_{-2}}{2}
$$

for $n \geq-1$. Employing (2.39), (2.40), (2.43), (2.44) in (2.31) and after some calculation, we obtain

$$
\begin{aligned}
x_{2 n}= & f^{-1}\left(\frac{\left(J_{2 n+1}+J_{2 n+1}^{\prime}\right) f\left(x_{0}\right)+\left(J_{2 n+1}-J_{2 n+1}^{\prime}\right) f\left(y_{0}\right)}{2}\right. \\
& \left.+\frac{\left(J_{2 n+2}-J_{2 n+2}^{\prime}\right) f\left(x_{-1}\right)+\left(J_{2 n+2}+J_{2 n+2}^{\prime}\right) f\left(y_{-1}\right)+b\left(J_{2 n}+J_{2 n}^{\prime}\right) f\left(x_{-2}\right)}{2}\right) \\
& \left.+\frac{b\left(J_{2 n}-J_{2 n}^{\prime}\right) f\left(y_{-2}\right)}{2}\right) \\
y_{2 n}=f^{-1} & \left(\frac{\left(J_{2 n+1}-J_{2 n+1}^{\prime}\right) f\left(x_{0}\right)+\left(J_{2 n+1}+J_{2 n+1}^{\prime}\right) f\left(y_{0}\right)}{2}\right. \\
& +\frac{\left(J_{2 n+2}+J_{2 n+2}^{\prime}\right) f\left(x_{-1}\right)+\left(J_{2 n+2}-J_{2 n+2}^{\prime}\right) f\left(y_{-1}\right)+b\left(J_{2 n}-J_{2 n}^{\prime}\right) f\left(x_{-2}\right)}{2} \\
& \left.+\frac{b\left(J_{2 n}+J_{2 n}^{\prime}\right) f\left(y_{-2}\right)}{2}\right), \\
x_{2 n+1} & =f^{-1}\left(\frac{\left(J_{2 n+2}-J_{2 n+2}^{\prime}\right) f\left(x_{0}\right)+\left(J_{2 n+2}+J_{2 n+2}^{\prime}\right) f\left(y_{0}\right)}{2}\right. \\
& +\frac{\left(J_{2 n+3}+J_{2 n+3}^{\prime}\right) f\left(x_{-1}\right)+\left(J_{2 n+3}-J_{2 n+3}^{\prime}\right) f\left(y_{-1}\right)}{2} \\
& \left.\quad \frac{b\left(J_{2 n+1}-J_{2 n+1}^{\prime}\right) f\left(x_{-2}\right)+b\left(J_{2 n+1}+J_{2 n+1}^{\prime}\right) f\left(y_{-2}\right)}{2}\right)
\end{aligned}
$$

and

$$
\begin{aligned}
y_{2 n+1}= & f^{-1}\left(\frac{\left(J_{2 n+2}+J_{2 n+2}^{\prime}\right) f\left(x_{0}\right)+\left(J_{2 n+2}-J_{2 n+2}^{\prime}\right) f\left(y_{0}\right)}{2}\right. \\
& +\frac{\left(J_{2 n+3}-J_{2 n+3}^{\prime}\right) f\left(x_{-1}\right)+\left(J_{2 n+3}+J_{2 n+3}^{\prime}\right) f\left(y_{-1}\right)}{2} \\
& \left.+\frac{b\left(J_{2 n+1}+J_{2 n+1}^{\prime}\right) f\left(x_{-2}\right)+b\left(J_{2 n+1}-J_{2 n+1}^{\prime}\right) f\left(y_{-2}\right)}{2}\right),
\end{aligned}
$$

for $n \geq-1$.

2.7. Case 7: $p_{n}=y_{n}, q_{n}=x_{n}, r_{n}=x_{n}, s_{n}=y_{n}$

In this case, system (1.8) is expressed as

$x_{n+1}=f^{-1}\left(a f\left(y_{n-1}\right)+b f\left(x_{n-2}\right)\right), y_{n+1}=f^{-1}\left(a f\left(x_{n-1}\right)+b f\left(y_{n-2}\right)\right)$, 
for $n \in \mathbb{N}_{0}$. Since $f$ is " $1-1$ ", from (2.49)

$$
f\left(x_{n+1}\right)=a f\left(y_{n-1}\right)+b f\left(x_{n-2}\right), f\left(y_{n+1}\right)=a f\left(x_{n-1}\right)+b f\left(y_{n-2}\right),
$$

for $n \in \mathbb{N}_{0}$. By using the change of variables

$$
f\left(x_{n}\right)=u_{n} \text {, and } f\left(y_{n}\right)=v_{n}, n \geq-2,
$$

system (2.50) is transformed to the following one

$$
u_{n+1}=a v_{n-1}+b u_{n-2}, \quad v_{n+1}=a u_{n-1}+b v_{n-2}, n \in \mathbb{N}_{0} .
$$

By summing the equations in (2.52) we get

$$
u_{n+1}+v_{n+1}=a\left(u_{n-1}+v_{n-1}\right)+b\left(u_{n-2}+v_{n-2}\right), n \in \mathbb{N}_{0},
$$

whereas by subtracting the second one from the first, we have

$$
u_{n+1}-v_{n+1}=-a\left(u_{n-1}-v_{n-1}\right)+b\left(u_{n-2}-v_{n-2}\right), n \in \mathbb{N}_{0} .
$$

From (2.5), we can write the solution of equation (2.53) as

$$
u_{n}+v_{n}=\left(u_{0}+v_{0}\right) J_{n+1}+\left(u_{-1}+v_{-1}\right) J_{n+2}+b\left(u_{-2}+v_{-2}\right) J_{n},
$$

for $n \geq-2$. On the other hand, by taking $a=0, b=-a, c=b$ in (1.4) and $S_{n}=J_{n+1}^{\prime}$, for all $n \geq-2$, which is called generalized Padovan sequence, in (1.5), from (2.54), we also have that

$$
u_{n}-v_{n}=\left(u_{0}-v_{0}\right) J_{n+1}^{\prime}+\left(u_{-1}-v_{-1}\right) J_{n+2}^{\prime}+b\left(u_{-2}-v_{-2}\right) J_{n}^{\prime},
$$

for $n \geq-2$. By summing the equations (2.55) and (2.56) we get

$$
\begin{aligned}
u_{n}= & \frac{J_{n+1}+J_{n+1}^{\prime}}{2} u_{0}+\frac{J_{n+1}-J_{n+1}^{\prime}}{2} v_{0}+\frac{J_{n+2}+J_{n+2}^{\prime}}{2} u_{-1} \\
& +\frac{J_{n+2}-J_{n+2}^{\prime}}{2} v_{-1}+b \frac{J_{n}+J_{n}^{\prime}}{2} u_{-2}+b \frac{J_{n}-J_{n}^{\prime}}{2} v_{-2}, n \geq-2 .
\end{aligned}
$$

By subtracting equation (2.56) from equation (2.55), we have

$$
\begin{aligned}
v_{n}= & \frac{J_{n+1}-J_{n+1}^{\prime}}{2} u_{0}+\frac{J_{n+1}+J_{n+1}^{\prime}}{2} v_{0}+\frac{J_{n+2}-J_{n+2}^{\prime}}{2} u_{-1} \\
& +\frac{J_{n+2}+J_{n+2}^{\prime}}{2} v_{-1}+b \frac{J_{n}-J_{n}^{\prime}}{2} u_{-2}+b \frac{J_{n}+J_{n}^{\prime}}{2} v_{-2}, n \geq-2 .
\end{aligned}
$$

From (2.51), (2.57) and (2.58) and after some calculation, we obtain

$$
\begin{aligned}
x_{n}= & f^{-1}\left(\frac{J_{n+1}+J_{n+1}^{\prime}}{2} f\left(x_{0}\right)+\frac{J_{n+1}-J_{n+1}^{\prime}}{2} f\left(y_{0}\right)\right. \\
& +\frac{J_{n+2}+J_{n+2}^{\prime}}{2} f\left(x_{-1}\right)+\frac{J_{n+2}-J_{n+2}^{\prime}}{2} f\left(y_{-1}\right) \\
& \left.+b \frac{J_{n}+J_{n}^{\prime}}{2} f\left(x_{-2}\right)+b \frac{J_{n}-J_{n}^{\prime}}{2} f\left(y_{-2}\right)\right), n \geq-2,
\end{aligned}
$$


and

$$
\begin{aligned}
y_{n}= & f^{-1}\left(\frac{J_{n+1}-J_{n+1}^{\prime}}{2} f\left(x_{0}\right)+\frac{J_{n+1}+J_{n+1}^{\prime}}{2} f\left(y_{0}\right)\right. \\
& +\frac{J_{n+2}-J_{n+2}^{\prime}}{2} f\left(x_{-1}\right)+\frac{J_{n+2}+J_{n+2}^{\prime}}{2} f\left(y_{-1}\right) \\
& \left.+b \frac{J_{n}-J_{n}^{\prime}}{2} f\left(x_{-2}\right)+b \frac{J_{n}+J_{n}^{\prime}}{2} f\left(y_{-2}\right)\right), n \geq-2 .
\end{aligned}
$$

2.8. Case 8: $p_{n}=x_{n}, q_{n}=y_{n}, r_{n}=y_{n}, s_{n}=x_{n}$

In this case, system (1.8) is written as in the form

$$
x_{n+1}=f^{-1}\left(a f\left(x_{n-1}\right)+b f\left(y_{n-2}\right)\right), y_{n+1}=f^{-1}\left(a f\left(y_{n-1}\right)+b f\left(x_{n-2}\right)\right),
$$

for $n \in \mathbb{N}_{0}$. Since $f$ is " $1-1$ ", from (2.61)

$$
f\left(x_{n+1}\right)=a f\left(x_{n-1}\right)+b f\left(y_{n-2}\right), f\left(y_{n+1}\right)=a f\left(y_{n-1}\right)+b f\left(x_{n-2}\right),
$$

for $n \in \mathbb{N}_{0}$. By using the change of variables

$$
f\left(x_{n}\right)=u_{n}, \text { and } f\left(y_{n}\right)=v_{n}, n \geq-2,
$$

system (2.62) is transformed to the following one

$$
u_{n+1}=a u_{n-1}+b v_{n-2}, \quad v_{n+1}=a v_{n-1}+b u_{n-2}, n \in \mathbb{N}_{0} .
$$

By summing the equations in (2.64) we get

$$
u_{n+1}+v_{n+1}=a\left(u_{n-1}+v_{n-1}\right)+b\left(u_{n-2}+v_{n-2}\right), n \in \mathbb{N}_{0},
$$

whereas by subtracting the second one from the first, we have

$$
u_{n+1}-v_{n+1}=a\left(u_{n-1}-v_{n-1}\right)-b\left(u_{n-2}-v_{n-2}\right), n \in \mathbb{N}_{0} .
$$

From (2.5), we can write the solution of equation (2.65) as

$$
u_{n}+v_{n}=\left(u_{0}+v_{0}\right) J_{n+1}+\left(u_{-1}+v_{-1}\right) J_{n+2}+b\left(u_{-2}+v_{-2}\right) J_{n},
$$

for $n \geq-2$. On the other hand, by taking $a=0, b=a, c=-b$ in (1.4) and $S_{n}=(-1)^{n} J_{n+1}$, for all $n \geq-2$, which is called generalized Padovan sequence, in (1.5), from (2.66), we also have that

$$
u_{n}-v_{n}=(-1)^{n}\left(\left(u_{0}-v_{0}\right) J_{n+1}-\left(u_{-1}-v_{-1}\right) J_{n+2}+b\left(u_{-2}-v_{-2}\right) J_{n}\right),
$$

for $n \geq-2$. From (2.68) we have

$$
u_{2 n}-v_{2 n}=\left(u_{0}-v_{0}\right) J_{2 n+1}-\left(u_{-1}-v_{-1}\right) J_{2 n+2}+b\left(u_{-2}-v_{-2}\right) J_{2 n},
$$

for $n \geq-1$ and

$$
u_{2 n+1}-v_{2 n+1}=-\left(u_{0}-v_{0}\right) J_{2 n+2}+\left(u_{-1}-v_{-1}\right) J_{2 n+3}-b\left(u_{-2}-v_{-2}\right) J_{2 n+1},
$$

for $n \geq-1$. From (2.67)

$$
u_{2 n}+v_{2 n}=\left(u_{0}+v_{0}\right) J_{2 n+1}+\left(u_{-1}+v_{-1}\right) J_{2 n+2}+b\left(u_{-2}+v_{-2}\right) J_{2 n},
$$


for $n \geq-1$. By summing the equations (2.69) and (2.71)

$$
u_{2 n}=u_{0} J_{2 n+1}+v_{-1} J_{2 n+2}+b u_{-2} J_{2 n}, n \geq-1 .
$$

By subtracting equation (2.69) from equation (2.71), we have

$$
v_{2 n}=v_{0} J_{2 n+1}+u_{-1} J_{2 n+2}+b v_{-2} J_{2 n}, n \geq-1 .
$$

From (2.67)

$$
u_{2 n+1}+v_{2 n+1}=\left(u_{0}+v_{0}\right) J_{2 n+2}+\left(u_{-1}+v_{-1}\right) J_{2 n+3}+b\left(u_{-2}+v_{-2}\right) J_{2 n+1},
$$

for $n \geq-1$. By summing the equations (2.70) and (2.74) we get

$$
u_{2 n+1}=v_{0} J_{2 n+2}+u_{-1} J_{2 n+3}+b v_{-2} J_{2 n+1}, n \geq-1 .
$$

By subtracting equation (2.70) from equation (2.74), we have

$$
v_{2 n+1}=u_{0} J_{2 n+2}+v_{-1} J_{2 n+3}+b u_{-2} J_{2 n+1}, n \geq-1 .
$$

From (2.63), (2.72), (2.73), (2.75), (2.76) and after some calculation, we obtain

$$
\begin{aligned}
x_{2 n} & =f^{-1}\left(f\left(x_{0}\right) J_{2 n+1}+f\left(y_{-1}\right) J_{2 n+2}+b f\left(x_{-2}\right) J_{2 n}\right), n \geq-1, \\
y_{2 n} & =f^{-1}\left(f\left(y_{0}\right) J_{2 n+1}+f\left(x_{-1}\right) J_{2 n+2}+b f\left(y_{-2}\right) J_{2 n}\right), n \geq-1, \\
x_{2 n+1} & =f^{-1}\left(f\left(y_{0}\right) J_{2 n+2}+f\left(x_{-1}\right) J_{2 n+3}+b f\left(y_{-2}\right) J_{2 n+1}\right), n \geq-1,
\end{aligned}
$$

and

$$
y_{2 n+1}=f^{-1}\left(f\left(x_{0}\right) J_{2 n+2}+f\left(y_{-1}\right) J_{2 n+3}+b f\left(x_{-2}\right) J_{2 n+1}\right), n \geq-1 .
$$

\section{Analytical Examples}

In this section, we give examples for Case 1, 4 and 7. Examples for the other cases can be constructed similarly.

Example 1. Let

$$
f(t)=t .
$$

Then, $D_{f}=\mathbb{R}$ and system (2.1) becomes

$$
x_{n+1}=a x_{n-1}+b x_{n-2}, \quad y_{n+1}=a y_{n-1}+b y_{n-2}, \quad n \in \mathbb{N}_{0} .
$$

Here we can also assume that parameters $a, b$ and initial values $x_{-2}, x_{-1}, x_{0}, y_{-2}, y_{-1}$, and $y_{0}$ are complex numbers, since function (3.1) is " $1-1$ " on $D_{f}=\mathbb{C}$. Function (3.1) is obviously an involution:

$$
f^{-1}(t)=f(t), \quad t \in D_{f}
$$

We see that formulas (2.7) and (2.8) hold. Using (3.1) in (2.7) and (2.8), we obtain that the general solution to system (3.2) is

$$
\begin{aligned}
x_{n} & =f^{-1}\left(f\left(x_{0}\right) J_{n+1}+f\left(x_{-1}\right) J_{n+2}+b f\left(x_{-2}\right) J_{n}\right) \\
& =x_{0} J_{n+1}+x_{-1} J_{n+2}+b x_{-2} J_{n}, n \geq-2,
\end{aligned}
$$




$$
\begin{aligned}
y_{n} & =f^{-1}\left(f\left(y_{0}\right) J_{n+1}+f\left(y_{-1}\right) J_{n+2}+b f\left(y_{-2}\right) J_{n}\right) \\
& =y_{0} J_{n+1}+y_{-1} J_{n+2}+b y_{-2} J_{n}, n \geq-2 .
\end{aligned}
$$

Example 2. Let

$$
f(t)=\frac{1}{t}
$$

Then $D_{f}=\mathbb{R} \backslash\{0\}$ and system (2.13) becomes

$$
x_{n+1}=\left(\frac{a}{x_{n-1}}+\frac{b}{x_{n-2}}\right)^{-1}, y_{n+1}=\left(\frac{a}{y_{n-1}}+\frac{b}{x_{n-2}}\right)^{-1}, n \in \mathbb{N}_{0} .
$$

Here we can also assume that parameters $a, b$ and initial values $x_{-2}, x_{-1}, x_{0}, y_{-1}$ and $y_{0}$ are complex numbers, since function (3.5) is " $1-1$ " on $D_{f}=\mathbb{C} \backslash\{0\}$.

Clearly, function (3.5) is an involution. We see that (2.22)-(2.24) hold. Using (3.5) in (2.22)-(2.24), we obtain the general solution to system (3.6):

$$
\begin{aligned}
x_{n} & =f^{-1}\left(f\left(x_{0}\right) J_{n+1}+f\left(x_{-1}\right) J_{n+2}+b f\left(x_{-2}\right) J_{n}\right) \\
& =\left(\frac{1}{x_{0}} J_{n+1}+\frac{1}{x_{-1}} J_{n+2}+\frac{b}{x_{-2}} J_{n}\right)^{-1} \\
& =\frac{x_{0} x_{-1} x_{-2}}{x_{-1} x_{-2} J_{n+1}+x_{0} x_{-2} J_{n+2}+b x_{0} x_{-1} J_{n}}, n \geq-2,
\end{aligned}
$$

$$
\begin{aligned}
y_{2 n+1}= & f^{-1}\left(f\left(x_{0}\right) J_{2 n+2}+f\left(x_{-1}\right)\left(J_{2 n+3}-a^{n+1}\right)+b f\left(x_{-2}\right) J_{2 n+1}+a^{n+1} f\left(y_{-1}\right)\right) \\
= & \left(\frac{1}{x_{0}} J_{2 n+2}+\frac{1}{x_{-1}}\left(J_{2 n+3}-a^{n+1}\right)+\frac{b}{x_{-2}} J_{2 n+1}+\frac{a^{n+1}}{y_{-1}}\right)^{-1} \\
= & \left(\frac{x_{-1} x_{-2} y_{-1} J_{2 n+2}+x_{0} x_{-2} y_{-1}\left(J_{2 n+3}-a^{n+1}\right)}{x_{0} x_{-1} x_{-2} y_{-1}}\right. \\
& +\frac{b x_{0} x_{-1} y_{-1} J_{2 n+1}+a^{n+1} x_{0} x_{-1} x_{-2}}{x_{0} x_{-1} x_{-2} y_{-1}}, n \geq-1, \\
y_{2 n+2}= & f^{-1}\left(f\left(x_{0}\right)\left(J_{2 n+3}-a^{n+1}\right)+f\left(x_{-1}\right) J_{2 n+4}+b f\left(x_{-2}\right) J_{2 n+2}+a^{n+1} f\left(y_{0}\right)\right) \\
= & \left(\frac{1}{x_{0}}\left(J_{2 n+3}-a^{n+1}\right)+\frac{1}{x_{-1}} J_{2 n+4}+\frac{b}{x_{-2}} J_{2 n+2}+\frac{a^{n+1}}{y_{0}}\right)^{-1} \\
= & \left(\frac{x_{-1} x_{-2} y_{0}\left(J_{2 n+3}-a^{n+1}\right)+x_{0} x_{-2} y_{0} J_{2 n+4}}{x_{0} x_{-1} x_{-2} y_{0}}\right. \\
& \left.+\frac{b x_{0} x_{-1} y_{0} J_{2 n+2}+a^{n+1} x_{0} x_{-1} x_{-2}}{x_{0} x_{-1} x_{-2} y_{0}}\right)^{-1}, n \geq-1 .
\end{aligned}
$$

Example 3. Let

$$
f_{k}(t)=t^{2 k+1}, k \in \mathbb{N}_{0}
$$


Then $D_{f_{k}}=\mathbb{R}$ and system (2.49) becomes

$$
x_{n+1}=\left(a y_{n-1}^{2 k+1}+b x_{n-2}^{2 k+1}\right)^{\frac{1}{2 k+1}}, y_{n+1}=\left(a x_{n-1}^{2 k+1}+b y_{n-2}^{2 k+1}\right)^{\frac{1}{2 k+1}}, n \in \mathbb{N}_{0} .
$$

Here we can also assume that parameters $a, b$ and initial values $x_{-2}, x_{-1}, x_{0}, y_{-2}, y_{-1}$, $y_{0}$ are complex numbers, since function (3.10) is " $1-1$ " on $D_{f_{k}}=\mathbb{C}$.

Function (3.10) is an involution:

$$
f_{k}^{-1}(t)=t^{\frac{1}{2 k+1}}, \quad t \in D_{f_{k}} \text {. }
$$

We see that (2.59) and (2.60) hold. Using (3.10) in (2.59) and (2.60), we obtain the general solution to system (3.11):

$$
\begin{aligned}
x_{n}= & f_{k}^{-1}\left(\frac{J_{n+1}+J_{n+1}^{\prime}}{2} f_{k}\left(x_{0}\right)+\frac{J_{n+1}-J_{n+1}^{\prime}}{2} f_{k}\left(y_{0}\right)+\frac{J_{n+2}+J_{n+2}^{\prime}}{2} f_{k}\left(x_{-1}\right)\right. \\
& \left.+\frac{J_{n+2}-J_{n+2}^{\prime}}{2} f_{k}\left(y_{-1}\right)+b \frac{J_{n}+J_{n}^{\prime}}{2} f_{k}\left(x_{-2}\right)+b \frac{J_{n}-J_{n}^{\prime}}{2} f_{k}\left(y_{-2}\right)\right) \\
= & \left(\frac{J_{n+1}+J_{n+1}^{\prime}}{2} x_{0}^{2 k+1}+\frac{J_{n+1}-J_{n+1}^{\prime}}{2} y_{0}^{2 k+1}+\frac{J_{n+2}+J_{n+2}^{\prime}}{2} x_{-1}^{2 k+1}\right. \\
& \left.+\frac{J_{n+2}-J_{n+2}^{\prime}}{2} y_{-1}^{2 k+1}+b \frac{J_{n}+J_{n}^{\prime}}{2} x_{-2}^{2 k+1}+b \frac{J_{n}-J_{n}^{\prime}}{2} y_{-2}^{2 k+1}\right)^{\frac{1}{2 k+1}},
\end{aligned}
$$

for $n \geq-2$,

$$
\begin{aligned}
y_{n}= & f_{k}^{-1}\left(\frac{J_{n+1}-J_{n+1}^{\prime}}{2} f_{k}\left(x_{0}\right)+\frac{J_{n+1}+J_{n+1}^{\prime}}{2} f_{k}\left(y_{0}\right)+\frac{J_{n+2}-J_{n+2}^{\prime}}{2} f_{k}\left(x_{-1}\right)\right. \\
& \left.+\frac{J_{n+2}+J_{n+2}^{\prime}}{2} f_{k}\left(y_{-1}\right)+b \frac{J_{n}-J_{n}^{\prime}}{2} f_{k}\left(x_{-2}\right)+b \frac{J_{n}+J_{n}^{\prime}}{2} f_{k}\left(y_{-2}\right)\right) \\
= & \left(\frac{J_{n+1}-J_{n+1}^{\prime}}{2} x_{0}^{2 k+1}+\frac{J_{n+1}+J_{n+1}^{\prime}}{2} y_{0}^{2 k+1}+\frac{J_{n+2}-J_{n+2}^{\prime}}{2} x_{-1}^{2 k+1}\right. \\
& \left.+\frac{J_{n+2}+J_{n+2}^{\prime}}{2} y_{-1}^{2 k+1}+b \frac{J_{n}-J_{n}^{\prime}}{2} x_{-2}^{2 k+1}+b \frac{J_{n}+J_{n}^{\prime}}{2} y_{-2}^{2 k+1}\right)^{\frac{1}{2 k+1}}
\end{aligned}
$$

for $n \geq-2$.

\section{ACKNOWLEDGEMENTS}

The authors are thankful to the editor and reviewers for their constructive review.

\section{REFERENCES}

[1] Y. Akrour, N. Touafek, and Y. Halim, "On a system of difference equations of second order solved in closed form," Miskolc Mathematical Notes, vol. 20, no. 2, pp. 701-717, 2019, doi: 10.18514/MMN.2019.2923. 
[2] A. M. Alotaibi, M. S. M. Noorani, and M. A. El-Moneam, "On the solutions of a system of thirdorder rational difference equations," Discrete Dynamics in Nature and Society, vol. 2018, 2018, doi: $10.1155 / 2018 / 1743540$.

[3] M. M. El-Dessoky and E. M. Elsayed, "On the solutions and periodic nature of some systems of rational difference equations," Journal of Computational Analysis and Applications, vol. 18, no. 2, pp. 206-218, 2015.

[4] H. El-Metwally, "Solutions form for some rational systems of difference equations," Discrete Dynamics in Nature and Society, vol. 2013, 2013, doi: 10.1155/2013/903593.

[5] E. M. Elabbasy and S. M. Eleissawy, "Qualitative properties for a higher order rational difference equation,” Fasciculi Mathematici, no. 50, pp. 33-50, 2013.

[6] E. M. Elabbasy, H. A. El-Metwally, and E. M. Elsayed, "Global behavior of the solutions of some difference equations," Advances in Difference Equations, vol. 2011, no. 1, pp. 1-16, 2011, doi: 10.1186/1687-1847-2011-28.

[7] E. M. Elabbasy and E. M. Elsayed, "Dynamics of a rational difference equation," Chinese Annals of Mathematics Series B, vol. 30, no. 2, pp. 187-198, 2009, doi: 10.1007/s11401-007-0456-9.

[8] E. M. Elsayed, "On the solutions and periodic nature of some systems of difference equations," International Journal of Biomathematics, vol. 7, no. 6, pp. 1-26, 2014, doi: 10.1142/S1793524514500673.

[9] E. M. Elsayed and A. M. Ahmed, "Dynamics of a three-dimensional systems of rational difference equations," Mathematical Methods in the Applied Sciences, vol. 39, no. 5, pp. 1026-1038, 2016, doi: $10.1002 / \mathrm{mma} .3540$.

[10] E. M. Elsayed, F. Alzahrani, I. Abbas, and N. H. Alotaibi, "Dynamical behavior and solution of nonlinear difference equation via Fibonacci sequence," Journal of Applied Analysis and Computation, vol. 10, no. 1, pp. 282-296, 2020, doi: 10.11948/20190143.

[11] Y. Halim and J. F. T. Rabago, "On the solutions of a second-order difference equation in terms of generalized Padovan sequences," Mathematica Slovaca, vol. 68, no. 3, pp. 625-638, 2018, doi: 10.1515/ms-2017-0130.

[12] M. Kara and Y. Yazlik, "Solvability of a system of nonlinear difference equations of higher order," Turkish Journal of Mathematics, vol. 43, no. 3, pp. 1533-1565, 2019, doi: 10.3906/mat-1902-24.

[13] A. Sanbo and E. M. Elsayed, "Analytical study of a system of difference equation," Asian Research Journal of Mathematics, vol. 14, no. 1, pp. 1-18, 2019, doi: 10.9734/arjom/2019/v14i130118.

[14] S. Stević, "On a two-dimensional solvable system of difference equations," Electronic Journal of Qualitative Theory of Differential Equations, vol. 2018, no. 104, pp. 1-18, 2018, doi: 10.14232/ejqtde.2018.1.104.

[15] S. Stević, B. Iričanin, and W. Kosmala, "Representations of general solutions to some classes of nonlinear difference equations," Advances in Difference Equations, vol. 2019, no. 1, pp. 1-21, 2019, doi: 10.1186/s13662-019-2013-8.

[16] S. Stević, B. Iricanin, W. Kosmala, and Z. Smarda, "Representation of solutions of a solvable nonlinear difference equation of second order," Electronic Journal of Qualitative Theory of Differential Equations, vol. 2018, no. 95, pp. 1-18, 2018, doi: 10.14232/ejqtde.2018.1.95.

[17] N. Taskara, D. T. Tollu, and Y. Yazlik, "Solutions of rational difference system of order three in terms of Padovan numbers," Journal of Advanced Research in Applied Mathematics, vol. 7, no. 3, pp. 18-29, 2015.

[18] D. T. Tollu, Y. Yazlik, and N. Taskara, "On fourteen solvable systems of difference equations," Applied Mathematics and Computation, vol. 233, pp. 310-319, 2014, doi: 10.1016/j.amc.2014.02.001.

[19] D. T. Tollu, Y. Yazlik, and N. Taskara, "On the solutions of two special types of Riccati difference equation via Fibonacci numbers," Advances in Difference Equations, vol. 2013, no. 1, pp. 1-7, 2013, doi: 10.1186/1687-1847-2013-174. 
[20] D. T. Tollu, Y. Yazlik, and N. Taskara, "On a solvable nonlinear difference equation of higher order," Turkish Journal of Mathematics, vol. 42, no. 4, pp. 1765-1778, 2018, doi: 10.3906/mat1705-33.

[21] N. Touafek and E. M. Elsayed, "On a second order rational systems of difference equations," Hokkaido Mathematical Journal, vol. 44, no. 1, pp. 29-45, 2015.

[22] Y. Yazlik and M. Kara, "On a solvable system of difference equations of higher-order with period two coefficients," Communications Faculty of Sciences University of Ankara Series A1 Mathematics and Statistics, vol. 68, no. 2, pp. 1675-1693, 2019, doi: 10.31801/cfsuasmas.548262.

[23] Y. Yazlik, D. T. Tollu, and N. Taskara, "On the solutions of difference equation systems with Padovan numbers," Applied Mathematics, vol. 4, no. 12A, pp. 15-20, 2013, doi: 10.4236/am.2013.412A1002.

[24] Y. Yazlik, D. T. Tollu, and N. Taskara, "On the solutions of a three-dimensional system of difference equations," Kuwait Journal of Science, vol. 43, no. 1, pp. 95-111, 2016.

Authors' addresses

M. Kara

Merve Kara, Karamanoglu Mehmetbey University, Kamil Ozdag Science Faculty, Department of Mathematics, 70100, Karaman, Turkey

E-mail address: mervekara@kmu.edu.tr

Y. Yazlik

(Corresponding author) Yasin Yazlik, Nevşehir Hacı Bektaş Veli University, Faculty of Science and Art, Department of Mathematics, 50300, Nevşehir, Turkey

E-mail address: yyazlikenevsehir.edu.tr 